\title{
Practical Position Tracking Control of a Robotic Manipulator Based on Fractional Order Sliding Mode Controller
}

\author{
Ahmet Dumlu \\ Department of Electrical \& Electronics Engineering, Faculty of Engineering and Architecture, \\ Erzurum Technical University, \\ Erzurum, Turkey \\ ahmetdumlu@erzurum.edu.tr
}

\begin{abstract}
In this study, the practical position tracking control of a robotic manipulator has been performed using the proposed fractional-order sliding mode control scheme (FOSMC). The designed control technique is composed by fractional calculus and sliding mode control (SMC) to guarantee the fast convergence of the joint positions to their desired values. The main idea behind the design of the FOSMC control is that, the sliding mode control composed with fractional calculus contributes to reach a robust control performance of a nonlinear system in presence of uncertainties, disturbances and un-modelled dynamics without a complete dynamic model of the system. To validate and demonstrate the performance of FO-SMC method, an industrial robot manipulator with external load has been used in the real time experimental studies. The experimental outcomes demonstrate that the proposed fractional-order sliding mode control strategy has a better trajectory tracking performance in presence of the external payload for tracking tasks compared with the classical SMC.
\end{abstract}

Index Terms-Fractional-order; Robot manipulator; Sliding mode control; Robot control.

\section{INTRODUCTION}

Robots, one of the most promising technologies, have been extensively used in many industrial areas such as manufacturing assembly [1], medical operations [2], drilling [3], transportation [4] and so on. However, the main areas of research in the field of robotic manipulators can be summarized as robotic manipulator design [5], optimum trajectory planning for industrial manipulators [6], [7], and robot manipulator control. Among these scientific research areas, the controlling of robotic manipulators has a crucial place to follow a precise and reliable trajectory between the initial and final position of their task.

As it is clearly known that, the robotic systems are timevarying nonlinear systems due to the nonlinear behavior of the system dynamics, modeling uncertainties, and external disturbances. Hence, achieving a good tracking performance of robotic systems is a difficult task. To overcome these undesirable uncertainties, various advanced control methods have been developed for different types of robotic systems such as; $\mathrm{H}$ infinity control [8], neural network control [9],

Manuscript received 3 January, 2018; accepted 7 April, 2018.
[10], adaptive fuzzy control [11], sliding mode control (SMC) [12], [13] and fractional-order control [14].

Among these control methods, fractional-order control strategy contributes to reach a robust control performance of a nonlinear system in presence of uncertainties, disturbances and un-modelled dynamics without a complete dynamic model of the system [15]. Fractional-order controllers use fractional order derivative and integral calculus that lead to more exibility and performance in the designing of the controller. Hence, in literature, fractional-order calculus is combined with other control methods to obtain more robust performance. N. Ullah et al. [16], has proposed a new fuzzy fractional order sliding mode control using the fractional calculus and then, the proposed method has been performed on servo actuation system. According to the obtained results, the proposed method has less tracking error compared to a classical SMC. S. Ebrahimkhani [17], has performed a novel robust fractional-order sliding mode controller with fractional order estimator for the doubly fed induction generator. The results have confirmed the robustness and the effectiveness of the proposed controller during variations of disturbances and parameters.

Another robust control technique used for trajectory tracking control of robotic system in literature is SMC method. SMC method is an effective and robust control technique and has a variable structure for the unknown dynamics of unknown loads and nonlinear systems. This control method is also used to simplify design, increase the trajectory tracking accuracy and reduce the model complexity [18]. The SMC technique provides a systematic approach that improves the system capability to eliminate the problem of stability preservation. The important aspect of a SMC is to control a nonlinear dynamic system subjected to the external disturbances or uncertainties by using a switching function which ensures the system states stay on the sliding surface [19], [20].

In literature, different types of SMC technique have been performed for robotic manipulator. J. Baek et al. [21] has proposed a sliding mode control for the two-DOF robot manipulator. Their experimental results have verified the high-precision tracking performance. The terminal sliding mode control for n-DOF robotic manipulator has been proposed by $\mathrm{Yu}$ et al. [22]. Second order terminal sliding 
mode controller for robotic manipulators has been performed in [23].

In this paper, motivated by the advantages of the $\mathrm{FO}$ calculus [15] and SMC, a FO sliding mode control scheme (FO-SMC) is proposed and performed on a real six DOF industrial robotic manipulator. The proposed control technique provides fast finite-time convergence of system state variable, strong robustness, and simple structure for implementation without using the information of the upper bounds on uncertainties and disturbances in the manipulator system. In order to illustrate the efficiency of FO-SMC, the real time studies have been realized and compared to the classical SMC under the external payload. The main contributions of this study are given as follows; Sliding mode controller (SMC) integrated with fractional calculus (FO) is developed to obtain strong robustness, fast finitetime convergence, precision, and chatter-free control. FOSMC is utilized to compensate the unknown system dynamics where no knowledge relating to the nonlinear model is available a priori. The use of real-time FO-SMC for trajectory tracking control of the nonlinear robotic system with unknown disturbances is novel.

The rest of the paper is organized as follows. Section II presents some preliminaries about FO calculus. The FOSMC for the n-DOF robotic manipulators has shown in Section III. In Section IV, the experimental outcomes have been demonstrated, followed by the conclusion in Section V.

\section{PREliminaries of FO CALCUlus}

In fractional calculus theory, the fractional order differentiator can be denoted by a general fundamental operator, $\mathcal{D}^{\alpha}$, with a non-integer $\alpha \in \mathfrak{R}$. Further, for $\alpha>0$, one obtains differentiators while $\alpha<0$ yields integrators.

In literature, several mathematical definitions have used to describe the fractional order integrals and derivatives. The most commonly used definition is the Riemann-Liouville (RL). As the Riemann-Liouville (RL) fractional derivative and integral of $\alpha_{t h}$ order of function $f(t)$ with respect to $t$ are given by [24]:

$$
\begin{gathered}
\mathcal{D}^{\alpha} f(t)=\frac{d^{\alpha} f(t)}{d t^{\alpha}}=\frac{1}{\Gamma(n-\alpha)}\left(\frac{d^{n}}{d t^{n}}\right)_{0}^{t} \frac{f(\tau)}{(t-\tau)^{\alpha+1-n}} d \tau, \\
\mathcal{D}^{-\alpha} f(t)=\mathcal{L}^{\alpha} f(t)=\frac{1}{\Gamma(\alpha)} \int_{0}^{t} \frac{f(\tau)}{(t-\tau)^{1-\alpha}} d \tau,
\end{gathered}
$$

where $n$ is an integer and $n-1 \leq \alpha<n$, while $\mathcal{D}^{\alpha}$ and $\mathcal{L}^{\alpha}$ denote the fractional derivative and integral, respectively. And $\Gamma($.$) is Euler's Gamma function given by$

$$
\Gamma(\alpha)=\int_{0}^{\infty} e^{-t} t^{\alpha-1} d t
$$

Additionally, the $n_{t h}$ order derivative $\left(d^{n} / d t^{n}\right)$ of the fractional derivative operator $\mathcal{D}^{\alpha} f(t)$ can be transformed as

$$
\frac{d^{n}}{d t^{n}}\left(\mathcal{D}^{\alpha} f(t)\right)=\mathcal{D}^{\alpha}\left(\frac{d^{n} f(t)}{d t^{n}}\right)=\mathcal{D}^{\alpha+n} f(t)
$$

\section{Fractional Order Sliding Mode Control Scheme}

A general $n$ - DOF robot manipulator's dynamics can be described as follows

$$
\tau=M(q) \ddot{q}+C(q, \dot{q}) \dot{q}+G(q)+F_{r}(q, \dot{q})+\tau_{d},
$$

where, $\ddot{q} \in \mathfrak{R}^{n}, \quad \dot{q} \in \mathfrak{R}^{n}$ and $q \in \mathfrak{R}^{n}$ are the angular acceleration, velocity and position of the manipulator's joints respectively, $M(q) \in \mathfrak{R}^{n \times n}$ is the symmetric positive definite inertia matrix, $\tau \in \mathfrak{R}^{n}$ is the control input torque of the joints, $C(q, \dot{q}) \in \mathfrak{R}^{n}$ expresses the Coriolis matrix, $G(q) \in \mathfrak{R}^{n}$ symbolizes the gravity force, $F_{r}(q, \dot{q}) \in \mathfrak{R}^{n}$ stands for the viscous and Coulomb friction force and finally $\tau_{d} \in \mathfrak{R}^{n}$ denotes the lumped disturbance. Representing (5) in a compact form yields

$$
\tau=M(q) \ddot{q}+H(q, \dot{q})+\tau_{d},
$$

where $H(q, \dot{q})=C(q, \dot{q}) \dot{q}+G(q)+F_{r}(q, \dot{q})$. If the both sides of (6) is multiplied by $M(q)^{-1}, \ddot{q}$ can be defined as follows

$$
\ddot{q}=f(q, \dot{q}, t)+g(q, t) u(t)+\xi(t, u(t)),
$$

where $f(q, \dot{q}, t)=-M(q)^{-1} H(q, \dot{q}), \quad g(q, t)=M(q)^{-1}$, $u(t)=\tau$ and $\xi(t, u(t))$ stands the bounded uncertainties of the system.

The aim of proposed control technique in this study is to control the robot manipulator's joint angles, $q(t)$, accurately for the given reference trajectory, $q_{d}(t)$. In order to meet the accurate trajectory tracking, the tracking error $e(t)=q_{d}(t)-q(t)$ should be minimized as much as possible.

In this study, the fractional order sliding mode control (FO-SMC) method has been used. The proposed FO-SMC block diagram for robotic manipulator can be seen in Fig. 1.

As shown in the block diagram, the proposed FO sliding control technique consists of two control parts and it can be defined mathematically by the following equation

$$
u(t)=u_{e q c l}(t)+u_{s w c l}(t)
$$

where $u_{\text {eqcl }}(t)$ is the equivalent control law and it can be obtained using a sliding surface function with an approximate model of the system and $u_{\text {swcl }}(t)$ is a switching discontinuous control law and it can be used to 
compensate the uncertainties affecting the system.

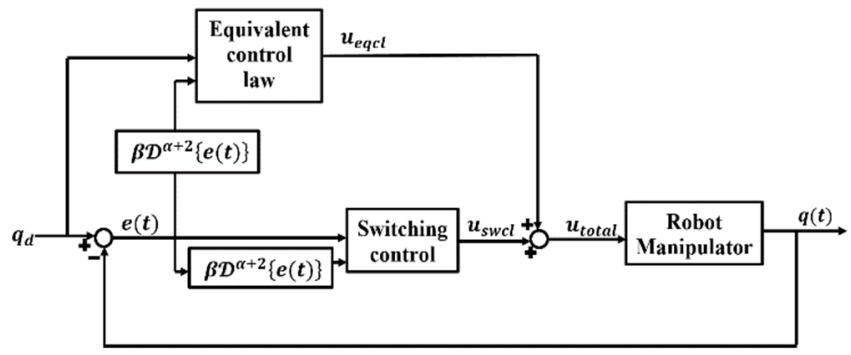

Fig. 1. The FO-SMC block diagram for robotic manipulator.

Sliding surface function is given as follows [25]

$$
s(t)=e(t)+\beta \int_{0}^{t} e(\tau) d \tau
$$

where $\beta$ is $n \times n$ diagonal positive definite control gains matrixes. To obtain fast finite-time convergence, strong robustness and precision control performance for a robot manipulator, the following FO-sliding surface function is designed by using FO calculus as follows

$$
s(t)=e(t)+\beta \mathcal{D}^{-\alpha}\{e(t)\}
$$

It is clear that if the value of $\alpha$ in (10) is taken as one, then (10) becomes (9). Two times differentiating (10) with respect to time, following equation is obtained as follows

$$
\ddot{s}(t)=\ddot{e}(t)+\beta \mathcal{D}^{-\alpha+2}\{e(t)\} .
$$

Additionally, using the second derivation of the tracking error function, (11) can be re-written as follows

$$
\ddot{s}(t)=\left(\ddot{q}_{d}-\ddot{q}\right)+\beta \mathcal{D}^{-\alpha+2}\{e(t)\} .
$$

Substituting $\ddot{q}$ from (7) into (12), yields

$$
\begin{gathered}
\ddot{s}(t)=\ddot{q}_{d}-f(t)-g(t) u(t)-\xi(t, u(t))+ \\
+\beta \mathcal{D}^{-\alpha+2}\{e(t)\} .
\end{gathered}
$$

In the sliding surface condition, if $s, \dot{s}$ and $\ddot{s}$ are equal to null, then the tracking error, $e(t)$, reaches to zero. Therefore, the equivalent control law $u_{\text {eqcl }}(t)$ can be obtained with uncertainties by using $\ddot{s}(t)=0$ as follows

$$
u_{e q c l}(t)=g(t)^{-1}\left(\begin{array}{l}
\ddot{q}_{d}-f(t)-\xi(t, u(t))+ \\
+\beta \mathcal{D}^{\alpha+2}\{e(t)\}
\end{array}\right) .
$$

In addition to this, it is not appropriate to use only the equivalent control law to control the system. As aforementioned above, the effect of the uncertainties which are constrained but unknown that can be serious for robotic system. Therefore, a switching control law, $u_{\text {swcl }}(t)$ in (14), should be added to control signal to ensure the robustness of robotic system against the external or internal disturbances. Because of the unknown term $\xi(t, u(t))$ is a physically bounded function, the switching control law $u_{s c l}(t)$ can be as designed follows

$$
u_{s w c l}(t)=g(t)^{-1}(\rho s+\mu \operatorname{sign}(\dot{s}))
$$

where $\rho$ and $\mu$ are $n \times n$ diagonal positive definite switching gain matrixes. Thus, the robot trajectory's total control law for the tracking is found as

$u(t)=g(t)^{-1}\left(\begin{array}{l}g(t)^{-1}\left(\begin{array}{l}\ddot{q}_{d}-f(t)-\xi(t, u(t))+ \\ +\beta \mathcal{D}^{\alpha+2}\{e(t)\} \\ +\rho s+\mu \operatorname{sign}(\dot{s})\end{array}\right)+\end{array}\right)$.

As it is shown in (16), if the switching gain increases, signum function can increase the chattering effect. To avoid this situation, the total feedback control law $\left(u_{\text {total }}\right)$ is given as follows

$$
\begin{aligned}
& u_{\text {total }}(t)= \\
& =g(t)^{-1}\left(\begin{array}{l}
g(t)^{-1}\left(\begin{array}{l}
\ddot{q}_{d}-f(t)-\xi(t, u(t))+ \\
+\beta \mathcal{D}^{\alpha+2}\{e(t)\} \\
+\rho s+\mu \operatorname{sat}(\dot{s})
\end{array}\right)+
\end{array}\right),
\end{aligned}
$$

where, $\operatorname{sat}(\dot{\sigma})=\left\{\begin{array}{cc}\operatorname{sign}(\dot{s}), & |\dot{s}|>\delta>0 \\ \frac{\dot{s}}{\delta}, & |\dot{s}| \leq \delta\end{array}\right\}$ and $\delta$ is a small arbitrary positive constant.

\section{EXPERIMENT}

\section{A. Experimental Setup}

The proposed control approach has been verified by performing experimental tests on a six-DOF industrial robot manipulator with end-effector tool as shown in Fig. 2. Robot consists of six rotational joints driven by six motors with encoders and six links. Table I shows DH parameters of the robot manipulator necessary to derive the kinematics of the robot. Detailed kinematics analysis can be found in [26].

TABLE I. DH PARAMETERS OF THE ROBOT MANIPULATOR.
\begin{tabular}{|c|c|c|c|c|}
\hline Link $i$ & $\theta_{i}$ & $d_{i}$ & $a_{i-1}$ & $\alpha_{i-1}$ \\
\hline 1 & $q_{1}$ & 0.125 & 0 & $\pi / 2$ \\
\hline 2 & $q_{2}+\pi / 2$ & 0 & 0.210 & 0 \\
\hline 3 & $q_{3}-\pi / 2$ & 0 & -0.075 & $-\pi / 2$ \\
\hline 4 & $q_{4}$ & 0.21 & 0 & $\pi / 2$ \\
\hline 5 & $q_{5}$ & 0 & 0 & $-\pi / 2$ \\
\hline 6 & $q_{6}$ & 0.07 & 0 & 0 \\
\hline
\end{tabular}

This manipulator has been controlled by using a PC based data acquisition device with six amplifiers and the controllers are commanding the motors and measuring the 
encoders at a rate of $1 \mathrm{kHz}$. In the experimental studies, an external payload has been placed on the end-effector tool to test the proposed controller performance and robustness against the uncertainties and un-modelled dynamics. The experiments have been carried out with Matlab/Simulink software by using Runge-Kutta solver with a fixed-step of 0.001 .

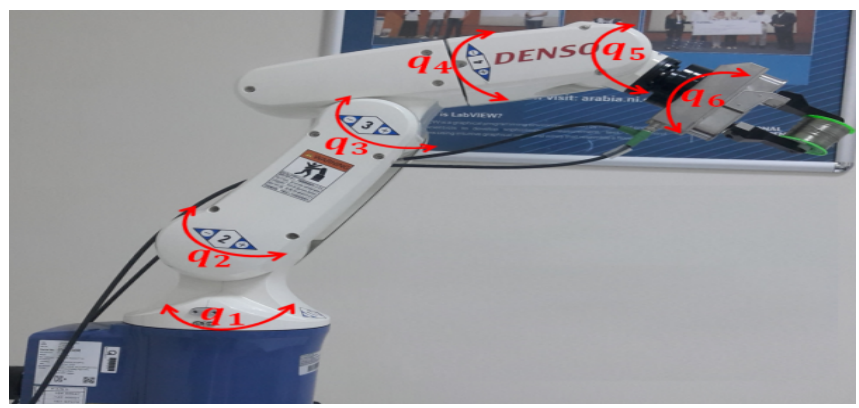

Fig. 2. Industrial robot manipulator used for the experimental tests.

\section{B. Experimental Results}

To demonstrate the performance and verify the effectiveness of the FO-SMC with respect to the classical SMC control for given tracking tasks, the following experiments have been performed.

As shown in Fig. 3, to analyse transient and steady-state responses of the controllers, the home position of the robot manipulator has been set up at $t=0$ as $P_{x_{i}}=-0.075 \mathrm{~m}$, $P_{y_{i}}=0 \mathrm{~m}, P_{z_{i}}=0.615 \mathrm{~m}$ and then a helix trajectory has been defined for the robot manipulator as shown in (18). The helix trajectory has an important characteristic to determine the controller' performances, because all the axis change continuously in the defined reference:

$$
\begin{cases}P_{x}=0.1485+0.0729 \cos (2 \pi 0.5 t) & \rightarrow 0<t \leq 12 \mathrm{~s} \\ P_{y}=0.1485+0.0729 \sin (2 \pi 0.5 t) & \rightarrow 0<t \leq 12 \mathrm{~s} \\ P_{z}=0.34-\frac{t}{100} \rightarrow & 0<t \leq 12 \mathrm{~s}\end{cases}
$$

Using the proposed FO-SMC and SMC control law, translational position tracking error of the end-effector has been shown in Fig. 4-Fig. 6. In this case, translational position tracking error of the end-effector is not directly measured, but the tracking error can be calculated from the kinematics of the robotic manipulator using the $\mathrm{DH}$ parameters as tabulated Table I.

As seen in Fig. 4-Fig. 6, the proposed FO-SMC a quite well tracking performance when compared to the SMC controller due to its FO actions for given tracking tasks. The higher error values at initial phase are occurred because of the difference between the home position and the initial position of the defined trajectory.

Additionally, to show the efficiency of the proposed FO$\mathrm{SMC}$, the root-mean-square-error (RMSE) values of the position tracking errors norm for both control approaches have been calculated based on the second period of the experimental data to show the steady-state control performance by using (19) and tabulated in Table II.

$$
e_{R M S E}=\sqrt{\frac{1}{N} \sum_{k=1}^{N} e(k)^{2}}
$$

where $N$ is equal to the number of sampling steps of the experiments.

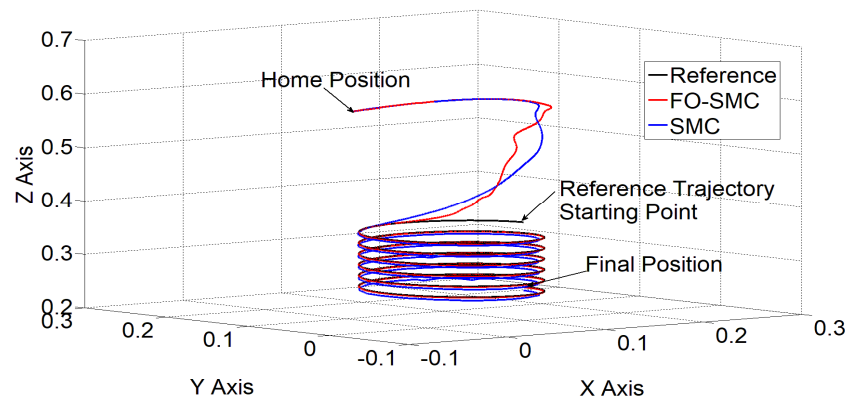

Fig. 3. Graphical representation of defined trajectory for robot manipulator.

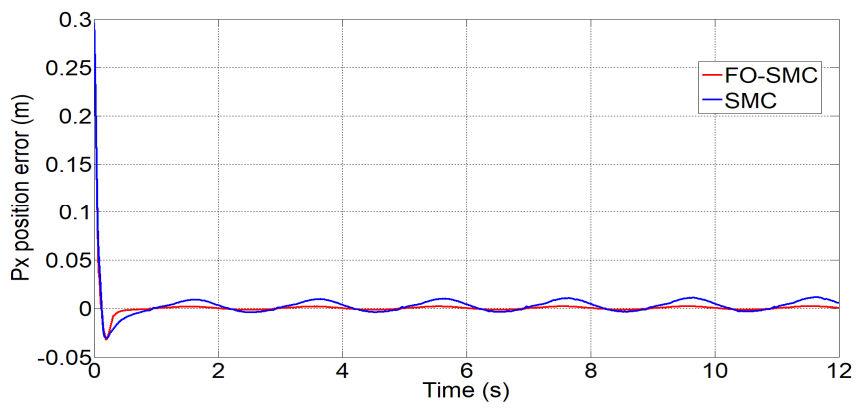

Fig. 4. $P_{x}$ position error of end-effector for defined trajectory.

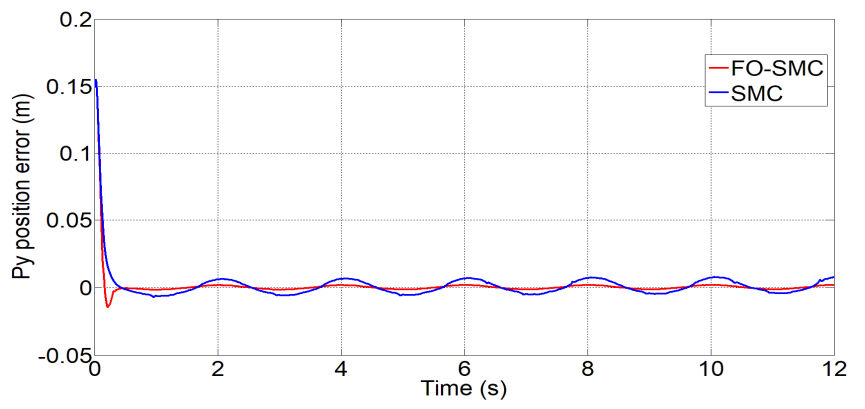

Fig. 5. $P_{y}$ position error of end-effector for defined trajectory.

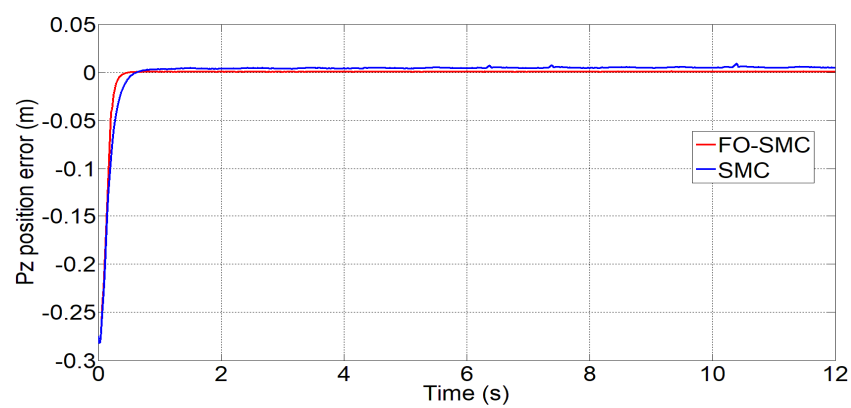

Fig. 6. $P_{z}$ position error of end-effector for defined trajectory.

TABLE II. TRANSLATIONAL POSITION TRACKING PERFORMANCE SUMMARY.

\begin{tabular}{|c|c|c|}
\hline Control Strategies & FO-SMC & SMC \\
\hline RMSE value of the $P_{x}$ tracking errors & 0.0014 & 0.0060 \\
\hline RMSE value of the $P_{y}$ tracking errors & 0.0011 & 0.0043 \\
\hline RMSE value of the $P_{z}$ tracking errors & 0.0009 & 0.0051 \\
\hline
\end{tabular}


As seen in Table I, RMSE values of $P_{x}, P_{y}$ and $P_{z}$ positions obtained using the proposed FO-SMC are less than those obtained using the classical SMC.

Addition to these, tracking performance of the manipulator joints with zoom-in tracking performances that provide desired position of the end-effector, tracking errors and their torque control signals for both controllers have been shown in Fig. 7-Fig. 11. Because of the 4th joint of the robot manipulator has no angular displacement for the given trajectory, tracking performance of the 4th joint has not been shown.

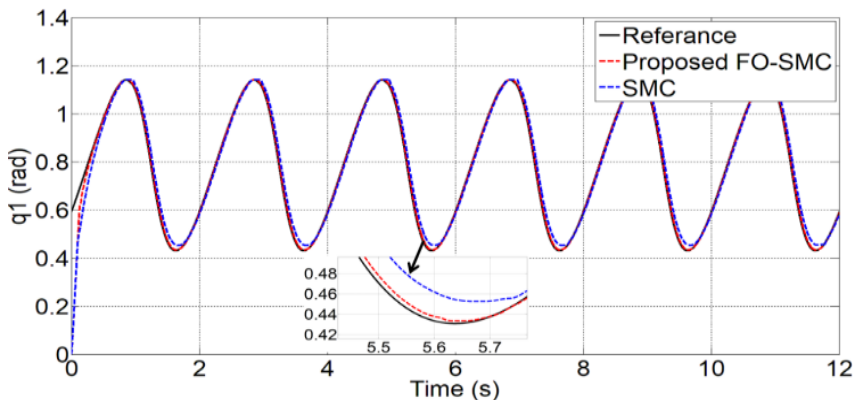

(a)

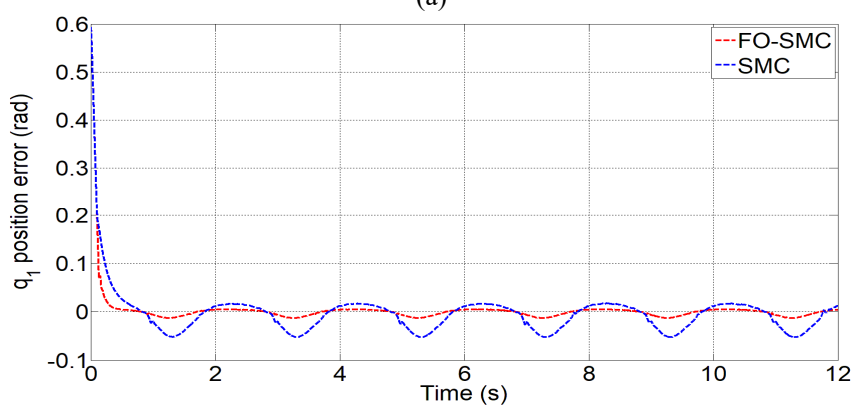

(b)

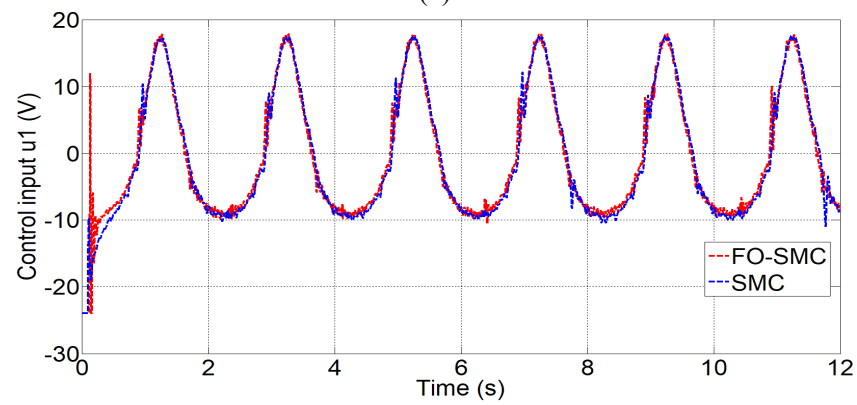

(c)

Fig. 7. (a) tracking performance of the 1 th joint $\left(q_{1}\right)$; (b) tracking error of the 1th joint $\left(q_{1}\right)$; (c) torque control input of the 1 th joint.

As can be seen in Fig. 7-Fig. 11(a-b), the classical SMC controller gave the poor tracking performance as compared to the FO-SMC due to its lack of fractional action against uncertainties and disturbances. Meanwhile, with the robust control designs, the FO-SMC improved the control performances with quite small tracking errors. By employing the fractional action to SMC, the controller makes the robotic system more robust against the presence of uncertainties and disturbances in the feedback loop. Additionally, both transient and steady-state error values are decreased by utilizing the FO-SMC controller. However, proposed controller has shorter settling time compared with the classical SMC controller. Hence, the proposed FO-SMC algorithm has provided better performance than the classical SMC algorithm with superior robustness and higher tracking accuracy.

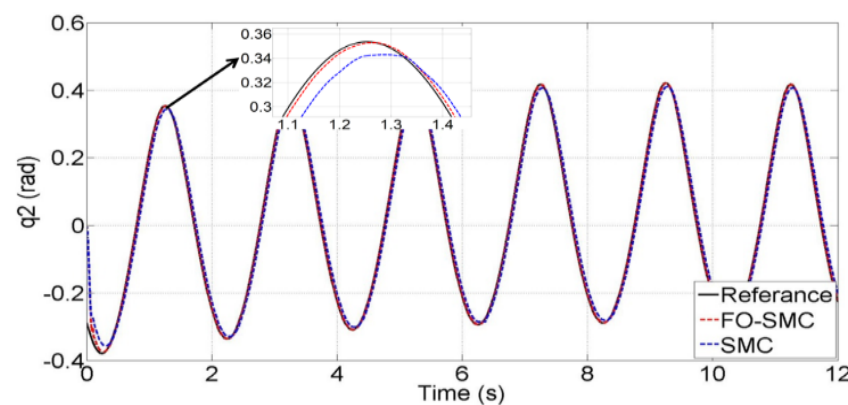

(a)

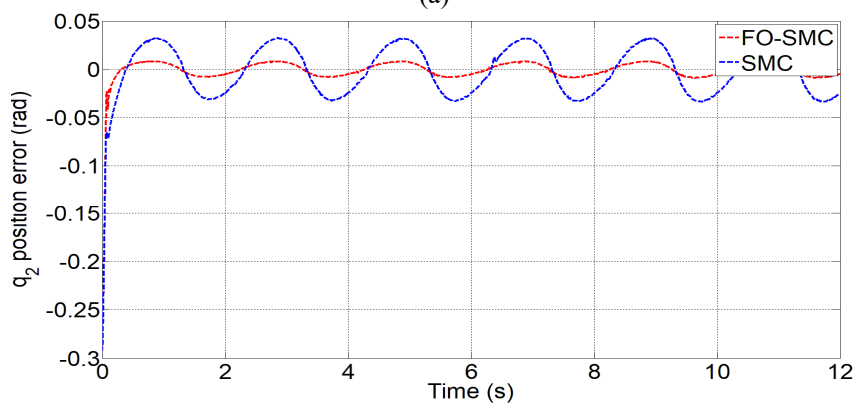

(b)

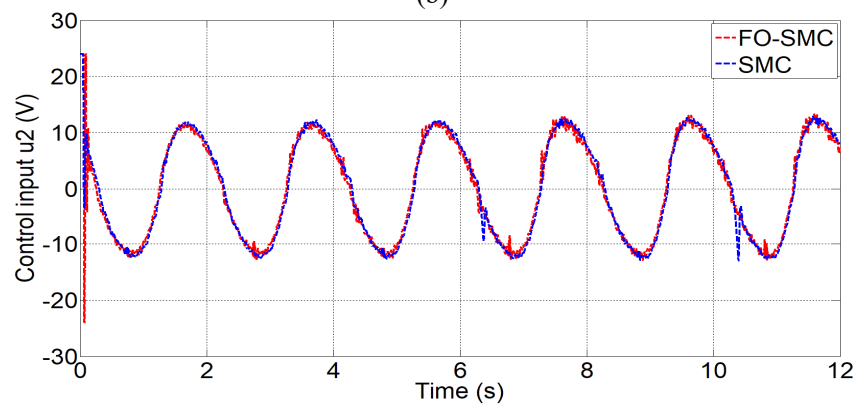

(c)

Fig. 8. (a) tracking performance of the 2 th joint $\left(q_{2}\right)$; (b) tracking error of the 2 th joint $\left(q_{2}\right)$; (c) torque control input of the 2 th joint.

Moreover, to show the steady-state control performance of the proposed FO-SMC with respect to the SMC controller, the RMSE values of the joint tracking error norms have been calculated based on the second period of the experimental data. And also, the integral of the square values (ISVs) of the torque control inputs for both control approaches have been calculated at the same period. The integral of the square values (ISVs) show the energy consumption and it can be calculated using (20). Obtained results have been tabulated in Tables III-IV.

TABLE III. RMSE VALUES OF THE JOINT TRACKING ERROR FOR BOTH CONTROL APPROACHES.

\begin{tabular}{|c|c|c|c|c|c|}
\hline $\begin{array}{c}\text { Control } \\
\text { Strategies }\end{array}$ & $\begin{array}{c}\Delta q_{1} \\
\text { (rad) }\end{array}$ & $\begin{array}{c}\Delta q_{2} \\
\text { (rad) }\end{array}$ & $\begin{array}{c}\Delta q_{3} \\
\text { (rad) }\end{array}$ & $\begin{array}{c}\Delta q_{5} \\
\text { (rad) }\end{array}$ & $\begin{array}{c}\Delta q_{6} \\
\text { (rad) }\end{array}$ \\
\hline $\begin{array}{c}\text { Classical } \\
\text { SMC } \\
\text { Control }\end{array}$ & 0.0258 & 0.0268 & 0.0335 & 0.0191 & 0.0257 \\
\hline $\begin{array}{c}\text { FO-SMC } \\
\text { Scheme }\end{array}$ & 0.0063 & 0.0060 & 0.0069 & 0.0032 & 0.0063 \\
\hline
\end{tabular}

TABLE IV. ISVS OF TORQUE CONTROL INPUTS.

\begin{tabular}{|c|c|c|c|c|c|}
\hline $\begin{array}{c}\text { Control } \\
\text { Strategies }\end{array}$ & $u_{1}(\mathbf{V})$ & $u_{2}(\mathbf{V})$ & $u_{3}(\mathbf{V})$ & $u_{5}(\mathbf{V})$ & $u_{6}(\mathbf{V})$ \\
\hline $\begin{array}{c}\text { Classical } \\
\text { SMC } \\
\text { Control }\end{array}$ & 525.7188 & 493.4640 & 448.1782 & 48.2697 & 512.1223 \\
\hline $\begin{array}{c}\text { FO-SMC } \\
\text { Scheme }\end{array}$ & 453.8477 & 388.8019 & 328.3401 & 28.4608 & 410.6390 \\
\hline
\end{tabular}




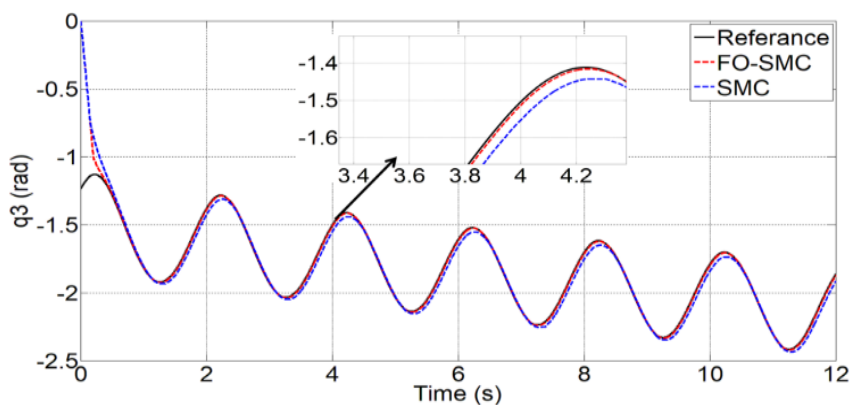

(a)

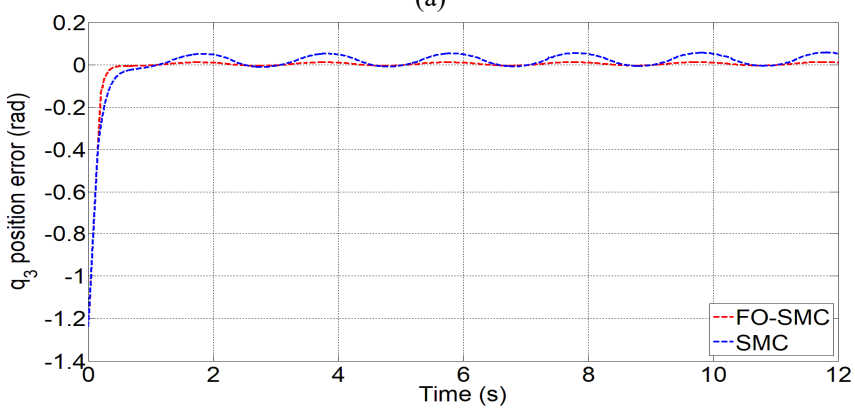

(b)

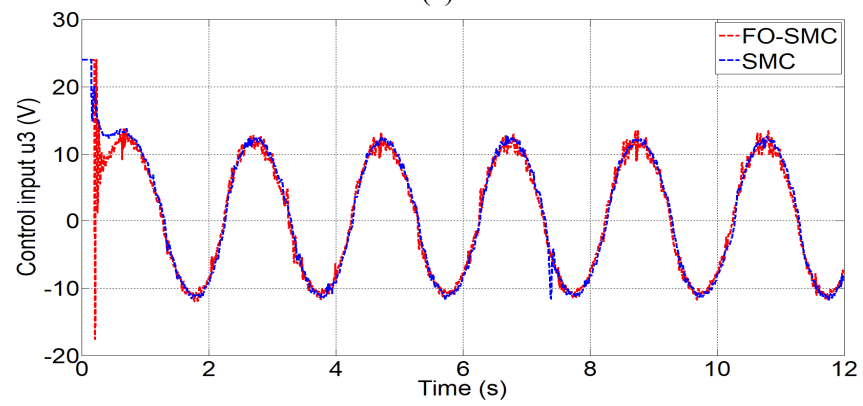

(c)

Fig. 9. (a) tracking performance of the 3th joint $\left(q_{3}\right)$; (b) tracking error of the 3 th joint $\left(q_{3}\right)$; (c) torque control input of the 3th joint.

As seen in Table III-Table IV, the RMSE values of the joint tracking error norms obtained using the proposed FOSMC are less than those obtained using the classical SMC. The lower energy consumption of torque control inputs are also achieved in the proposed FO-SMC scheme.

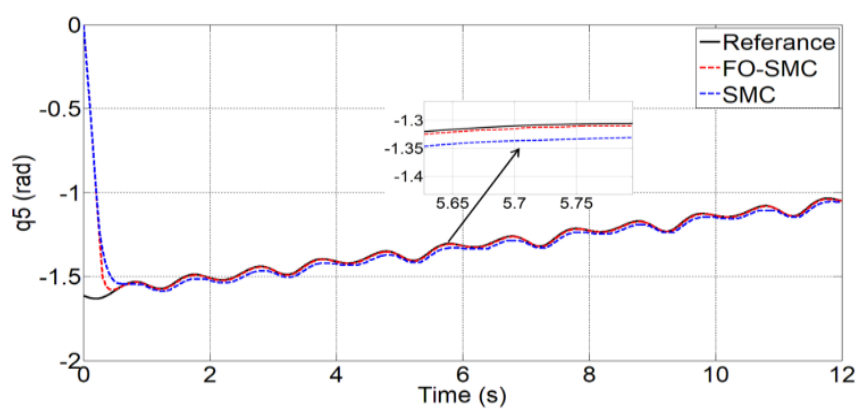

(a)

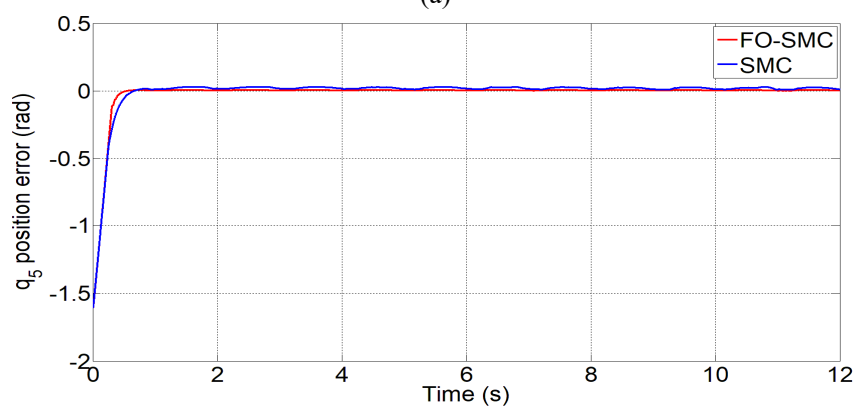

(b)

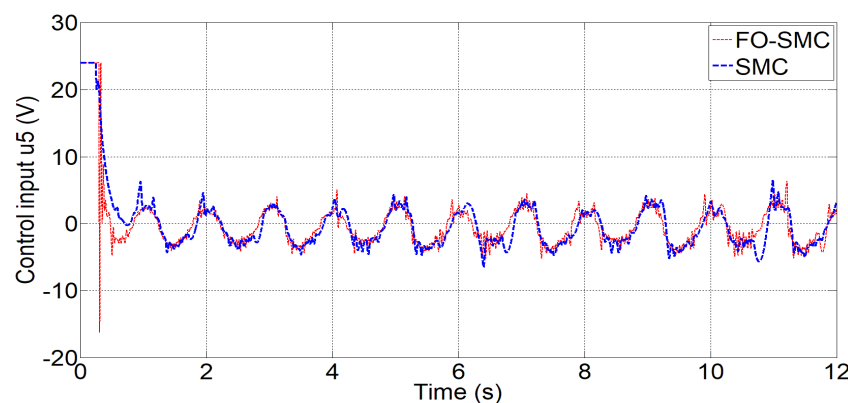

(c)

Fig. 10. (a) tracking performance of the 5th joint $\left(q_{5}\right)$; (b) tracking error of the 5 th joint $\left(q_{5}\right)$; (c) torque control input of the 5 th joint.

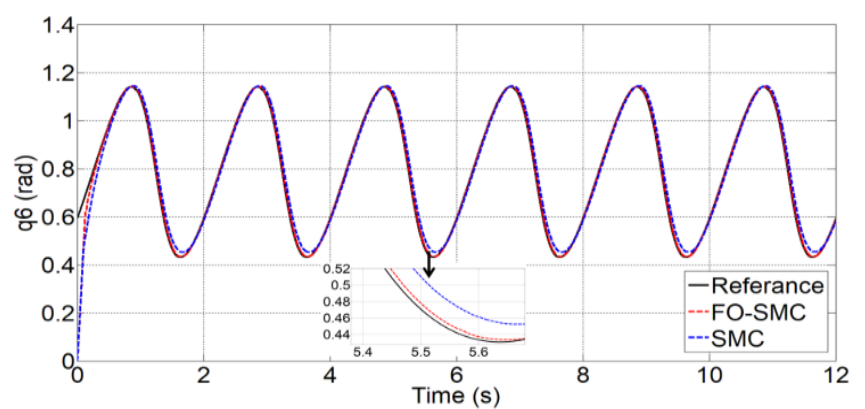

(a)

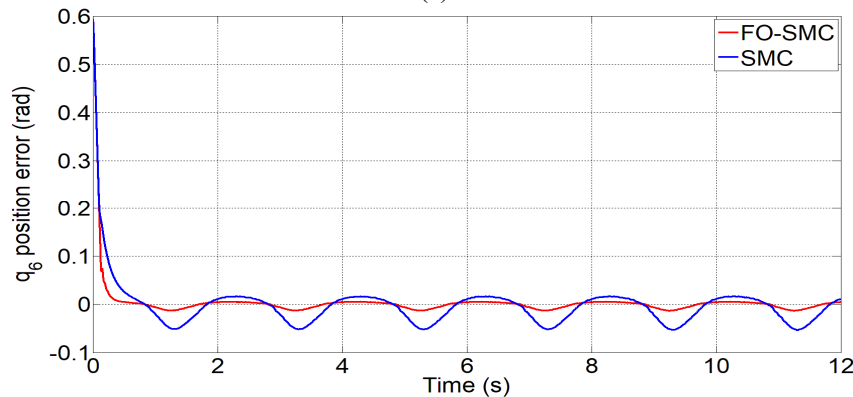

(b)

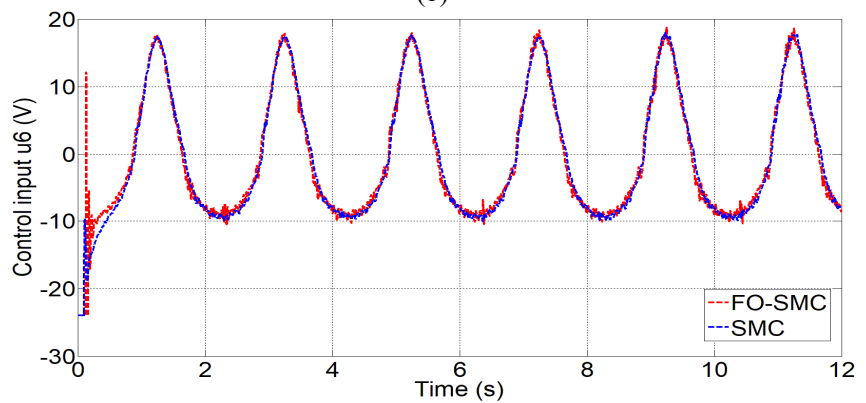

(c)

Fig. 11. (a) tracking performance of the 6th joint $\left(q_{6}\right)$; (b) tracking error of the 6th joint $\left(q_{6}\right)$; (c) torque control input of the 6th joint.

Also, the torque control inputs of proposed FO-SMC are little noisy but bounded without any noticeable control chattering

$$
I S V=\int_{0}^{t} V_{i}^{2}(u) d u
$$

\section{CONCLUSIONS}

In this study, the proposed FO-SMC method has been presented and validated on a real six-DOF robotic manipulator to show its strong robustness, precision, and chatter-free control. To achieve finite-time convergence, better tracking of trajectory and chattering-free control inputs, the fractional calculus has combined with the sliding 
mode control. A well-tuned classical sliding mode controller is also performed to the robotic manipulator to show the superiority of the proposed FO-SMC.

The experimental results clearly show that the proposed FO-SMC method has performed effectively in perspective of rapid response, fast finite-time convergence, significant improvements in terms of minimizing the trajectory tracking error when it compared to the responses of the classical SMC. According to obtained the average position tracking performance of the all joints, the steady-state error value of the classical SMC is greater than about three times that of the fractional-order SMC controller. In addition to these, the control inputs of proposed FO-SMC are little noisy but bounded without any noticeable control chattering. Also, lower energy consumption with average $20 \%$ improvement of control inputs is achieved in the proposed FO-SMC scheme compared to the classical SMC. For these reasons, it can be said that the FO-SMC controller is more robust than the classical SMC controller to perform tracking tasks.

For future work, adaptive fractional order sliding mode control or fractional-order sliding mode controller with fractional order estimator will be performed for n-DOF robotic manipulators. With the aid of the adaptive fractional order controller or fractional order estimator, it is expected that the trajectory tracking error value can be remarkably reduced.

\section{REFERENCES}

[1] B. Sadrfaridpour, H. Saeidi, Y. Wang, "An integrated framework for human-robot collaborative assembly in hybrid manufacturing cells", 2016 IEEE Int. Conf. Automation Science and Engineering (CASE 2016), Fort Worth, TX, 2016, pp. 462-4670. DOI: 10.1109/COASE.2016.7743441.

[2] Y. Chen et al., "Safety-enhanced motion planning for flexible surgical manipulator using neural dynamics", in IEEE Trans. Control Systems Technology, vol. 25, no. 5, pp. 1711-1723, 2017. DOI: 10.1109/TCST.2016.2628806.

[3] S. Fani, et al., "Simplifying telerobotics: wearability and teleimpedance improves human-robot interactions in teleoperation", in IEEE Robotics \& Automation Magazine, vol. 25, no. 1, pp. 77-88, 2018. DOI: 10.1109/MRA.2017.2741579.

[4] J. Wu, X. Yue, W. Li, "Integration of hardware and software designs for object grasping and transportation by a mobile robot with navigation guidance via a unique bearing-alignment mechanism", in IEEE/ASME Trans. Mechatronics, vol. 21, no. 1, pp. 576-583, 2016. DOI: 10.1109/TMECH.2015.2429681.

[5] N. Zemiti, G. Morel, T. Ortmaier, N. Bonnet, "Mechatronic design of a new robot for force control in minimally invasive surgery", in IEEE/ASME Trans. Mechatronics, vol. 12, no. 2, pp. 143-153, 2007. DOI: 10.1109/TMECH.2007.892831.

[6] K. K. Ayten, M. N. Sahinkaya, A. Dumlu, "Real time optimum trajectory generation for redundant/hyper-redundant serial industrial manipulators", in Int. Journal of Advanced Robotic Systems, pp. 1-14, 2017. DOI: $10.1177 / 1729881417737241$.

[7] K. K. Ayten, M. N. Sahinkaya, A. Dumlu, "Optimum trajectory generation for redundant/hyper-redundant manipulators", in IFACPapersOnLine, vol. 49, no. 21, pp. 493-500, 2016. DOI: 10.1016/j.ifacol.2016.10.651.

[8] M. Makarov, M. Grossard, P. Rodriguez-Ayerbe, D. Dumur, "Modeling and preview $\mathrm{H}$ infty control design for motion control of elastic-joint robots with uncertainties", in IEEE Trans. Industrial Electronics, vol. 63, no. 10, pp. 6429-6438, 2016. DOI:
10.1109/TIE.2016.2583406.

[9] C. Sun, H. Gao, W. He, Y. Yu, "Fuzzy neural network control of a flexible robotic manipulator using assumed mode method", in IEEE Trans. Neural Networks and Learning Systems, pp. 1-14. DOI: 10.1109/TNNLS.2017.2743103.

[10] N. Nikdel, P. Nikdel, M. A. Badamchizadeh, I. Hassanzadeh, "Using neural network model predictive control for controlling shape memory alloy-based manipulator", in IEEE Trans. Industrial Electronics, vol. 61, no. 3, pp. 1394-1401, 2014. DOI: 10.1109/TIE.2013.2258292.

[11] H. C. Huang, "Fusion of modified bat algorithm soft computing and dynamic model hard computing to online self-adaptive fuzzy control of autonomous mobile robots", in IEEE Trans. Industrial Informatics, vol. 12, no. 3, pp. 972-979, 2016. DOI: 10.1109/TII.2016.2542206.

[12] A. Dumlu, K. Erenturk, A. Kaleli, K. K. Ayten, “A comparative study of two model-based control techniques for the industrial manipulator", in Robotica, vol. 35, no. 10, pp. 2036-2055, 2016. DOI: $10.1017 / \mathrm{S} 0263574716000709$.

[13] A. Basci, K. Caan, K. Orman, A. Derdiyok, "Trajectory tracking control of a four rotor unmanned aerial vehicle based on continuous sliding mode controller", Elektronika ir Elektrotechnika, vol. 23, no. 3, pp. 12-19, 2017. DOI: 10.5755/j01.eie.23.3.18325.

[14] J. Viola, L. Angel, "Identification, control and robustness analysis of a robotic system using fractional control", in IEEE Latin America Trans., vol. 13, no. 5, pp. 1294-1302, 2015. DOI: 10.1109/TLA.2015.7111982.

[15] D. Valerio, J. S. Costa, "Introduction to single-input, single-output fractional control", in IET Control Theory \& Applications, vol. 5, no. 8, pp. 1033-1057, 2011. DOI: 10.1049/iet-cta.2010.0332.

[16] N. Ullah, W. Shaoping, M. I., Khattak, M. Shafi, "Fractional order adaptive fuzzy sliding mode controller for a position servo system subjected to aerodynamic loading and nonlinearities", in Aerospace Science and Technology, vol. 43, pp. 381-387, 2015. DOI: 10.1016/j.ast.2015.03.020.

[17] S. Ebrahimkhani, "Robust fractional order sliding mode control of doubly-fed induction generator (DFIG)-based wind turbines", in ISA Trans., vol. 63, pp. 343-354, 2016. DOI: 10.1016/j.isatra.2016.03.003.

[18] V. Utkin, "Variable structure systems with sliding modes", in IEEE Trans. Automatic Control, vol. 22, no. 2, pp. 212-222, 1977. DOI: 10.1109/TAC.1977.1101446.

[19] S. H. Zak, S. Hui, "On variable structure output feedback controllers for uncertain dynamic systems", in IEEE Trans. Automatic Control, vol. 38 , no. 10, pp. 1509-1512, 1993. DOI: 10.1109/9.241564.

[20] C. Edwards, S. K. Spurgeon, "Sliding mode stabilization of uncertain systems using only output information", in International Journal of Control, vol. 65, no. 5, pp. 1129-1144, 2001. DOI: $10.1080 / 00207179508921587$.

[21] J. Baek, M. Jin, S. Han, "A new adaptive sliding-mode control scheme for application to robot manipulators", in IEEE Trans. Industrial Electronics, vol. 63, no. 6, pp. 3628-3637, 2016. DOI: 10.1109/TIE.2016.2522386.

[22] S. Yu, X. Yu, B. Shirinzadeh, "Continuous finite-time control for robotic manipulators with terminal sliding mode control", in Automatica, vol. 41, no. 11, pp. 1957-1964, 2005. DOI: 10.1016/j.automatica.2005.07.001.

[23] S. Mondal, C. Mahanta, "Adaptive second order terminal sliding mode controller for robotic manipulators", J. Franklin Inst., vol. 351, no. 4, pp. 2356-2377, 2014. DOI: 10.1016/j.jfranklin.2013.08.027.

[24] S. Das, Functional Fractional Calculus for System Identification and Controls. Springer, 2008.

[25] Chih-Min Lin, C. F. Hsu, "Self-learning fuzzy sliding-mode control for antilock braking systems", in IEEE Trans. Control Systems Technology, vol. 11, no. 2, pp. 273-278, 2003. DOI: 10.1109/TCST.2003.809246.

[26] Y. S. Yong, Y. Huang, R. Chiba, T. Arai, T. Ueyama, J. Ota, "Teaching-playback robot manipulator system in consideration of singularities", IEEE/ASME Int. Conf. Advanced Intelligent Mechatronics, Wollongong, NSW, 2013, pp. 453-458. DOI: 10.1109/AIM.2013.6584133. 